investigators and rebutted by other workers; the secret of malignancy has been attributed to chromosome abnormalities, to gene mutation, lack of hormone balance, irregularity of enzyme constitution, etc.

The concept submitted below is possibly little more than an integration of current ideas, but it is set forth because this simple chemical aspect has stimulated us to what we believe to be intelligently planned if, thus far, unfruitful experiments. It may occasion useful criticism and clarification by others who can bring to bear upon it diverse aspects of knowledge.

The essence of the idea is the formulation of a molecule in the chromosome capable of splitting into two similar symmetrical portions. Visualization of the chromosome gives a picture of the vital process of reconstitution of this most important nuclear material in each 'daughter' cell. Further, in the case of the malignant cell, the molecule is to be conceived as impelled on a course of irreversible division. In the chromosome itself, no doubt associated with other nuclear constituents, there would the severance of this molecule during division of

ceeds directly and continually instead of via the usual attachment of the metabolic type of nucleotide groups.

The factors determining the abnormal production of nuclear material might, therefore, be various, that is to say, any of the agencies which we term carcinogenic; for example, the effects occasioned by the chemical or physico-chemical attachment of the well-known carcinogenic chemicals or their reaction products, by radioactive elements, X-rays or light waves, or more directly by a virus which in itself is a compound tetra-nucleotide which undergoes division with little 'metabolic' activity.

On this hypothesis the rapidity with which a virus may induce tumours in certain organisms compared with the slow influence of pure chemicals and hormones would be explicable, while the apparent anticancer influence ascribed to some vitamins of the $B$ complex and also the role of co-carcinogens could be interpreted in terms of chemical structure. The disappearance or alteration of normal cell function in many cases could also be inferred, while the occurrence of simple tumours and various grades of malignancy can all be comprehended as governed by such a mechanism operating through the pyrophosphoric di-nucleic acid structure.

Recently Stedman and Sted$\operatorname{man}^{1}$ have described a protein component of cell nuclei present in much greater proportion than nucleic acid, but the discovery

Purine - Ribose - Phosphate - Phosphate - Ribose - Purine does not necessarily overthrow the belief that nucleic
acid is the master constituent. The chromosomin moiety may play a most important part in providing the requisite environment for the elaboration of the 'double' nucleic acid.

\section{H. Donovan.}

D. L. WOODHOUSE.

Cancer Research Laboratory,

Medical School, Hospitals Centre,

Birmingham, 15. Bept. 29. (co-carboxylase), riboflavine-adenine dinucleotide, and phosphagen, while the active principle of embryonic extract which promotes growth of cultured tissue, and possibly the milk factor of inherited mammary cancer in mice, may possess this structure. Consideration of the behaviour of viruses, which have attributes of a living organism and of a pure chemical, gives support to such an idea. It has been suggested that when such an entity multiplies, it does so by laying down a copy of itself on a "template' system.

We suggest, then, that in the 'resting' phase, that is, when engaged in ordinary cellular activities, the cell has, as its essential basis, molecules of nucleic acid joined by pyrophosphoric linkings to nucleotides similar to those mentioned above which subserve its normal metabolic activity. When cell division is about to take place the metabolic nucleotides on one side of the linkage are replaced by, or are converted into, nucleotides which are the mirror image of those on the primary nucleic acid portion. The symmetrical, double nucleic acid then splits, with resultant division of the chromosomes accompanied by the associated phenomena which comprise 'cell division'.

In the case of the malignant cell, however, we would postulate that because of the abnormal juxtaposition of specific side chains, or the presence of abnormal linkings in such groups, the symmetrical development of the dual nucleic-acid molecule pro-
1 Stedman, E., and Stcdman, Mrs. E., Natuke, 152, 267 (1943).

\section{Flinders Petrie's Racial Types} report of a committee which had onabled the late Sir Flinders Petrie to photograph and make casts of a large series of 'Racial Types' from Egyptian wall-reliefs and frescoes. A limited edition of prints was published and has become very rare. In the report of the committee it was stated that the negatives were deposited with Mr. Browning Hogg, 75 High Street, Bromley, Kent, who would supply prints. It has been ascertained that Mr. Hogg ceased to live in Bromley after 1888; but the Association has no record concerning the negatives subsequent to 1887. It is now desired to trace them if by any means possible, since a number of these 'Racial Typos' either are not included in Max Burchardt's series (c. 1913) or are much better photographs, and no later work of this nature has been done. The Association will be grateful for any information.

British Association

for the Advancement of Science, (Secretary.)
IN 1887, the British Association published the

O. J. R. Howarth. London, W.1. 